\title{
CORRELATION OF TUNED FREE VIBRATION CHARACTERISTICS WITH MISTUNING SENSITIVITY FOR A BLADED DISK
}

\author{
Mahmoud I. Hussein ${ }^{*}$, Christophe Pierre ${ }^{\dagger}$, and Matthew P. Castanier ${ }^{\ddagger}$ \\ The University of Michigan \\ Ann Arbor, MI 48109-2125
}

\begin{abstract}
The inevitable occurrence of blade mistuning in turbomachinery rotors is known to cause vibration localization among the blades. This in turn increases the likelihood of an excessive vibration response at certain conditions, thus posing a major safety concern. This problem motivates the exploration of the various factors that affect the degree to which the response of a bladed disk increases as a result of mistuning. This paper shows that there is a correlation between the tuned system free vibration characteristics and the mistuned system forced response. More specifically, there is a three-way link among (1) the properties of the tuned structure eigenfrequency curves, (2) physical notions such as disk/blade participation and structural interblade coupling, and (3) the sensitivity of the blades' response to mistuning. These correlations are established by comparing a large set of mistuned forced response data to the tuned free vibration characteristics for a model of an industrial bladed disk. The forced response calculations are obtained using a reduced order modeling methodology. ${ }^{1-3}$ Although the results presented pertain specifically to the bladed disk considered, some of the ideas and conclusions could be extended to bladed disk structures in general. On this basis, some guidelines are presented that could serve as an inexpensive design tool for assessing mistuning sensitivity for bladed disks solely relying on tuned free response information.
\end{abstract}

\section{INTRODUCTION}

In practice, the individual sectors that comprise a turbomachinery rotor are seldom identical. There are always small variations in the structural properties of

Copyright (C) 2003 by Mahmoud I. Hussein. Published by the American Institute of Aeronautics and Astronautics, Inc., with permission.

*Graduate Student Research Assistant, Department of Mechanical Engineering.

†Professor, Dept. of Mechanical Engineering. Senior Member AIAA.

*Associate Research Scientist, Dept. of Mechanical Engineering. Senior Member AIAA. individual blades, which may be attributed to manufacturing and material tolerances or in-service degradadation. These blade-to-blade variations are commonly referred to as blade mistuning. Since a bladed disk is a periodic structure (Figure 1a), these small irregularities may inhibit the even distribution of vibration energy among the blades, and result in vibration localization. This phenomenon is a major source of safety concern because in some cases vibration energy may be confined to only a few blades, thus resulting in excessive deflections. Localization has attracted considerable interest in solid state physics, ${ }^{4-7}$ and later in the structural dynamics field..$^{1-3,8-14}$

The simplest approach to predict the extent of localization, and its effect on the overall response, is to solve for the forced response of the entire mistuned system for every possible irregular configuration. Such route entails enormous computational effort for obvious reasons. Recently an accurate and efficient reduced order modeling technique has been developed for this problem. ${ }^{1-3}$ The technique utilizes cyclic symmetry, component mode synthesis, and statistical methods to predict the mistuned forced response. This methodology promises to be a highly effective tool for predicting mistuning sensitivity (MS), which is defined here as the ratio of the maximum mistuned forced response amplitude to that of the tuned system.

On another track, there are ongoing efforts to establish a method for predicting mistuning sensitivity solely from information describing the tuned free vibration characteristics and the operation conditions. If such a connection and the associated relationships are thoroughly understood, an inexpensive design tool for early assessments of mistuning sensitivity can be provided. The aim of this paper is to establish these relationships using exhaustive numerical simulations.

The tuned free vibration characteristics can be derived from an eigenfrequency versus number of nodal diameters plot, such as the one displayed in Figure 2. The number of nodal diameters refers to the lines of zero displacement across the diameter of the disk. Recently Bladh et al. devised a method to calculate eigenfrequencies for interblade phase angles that are between those corresponding to integer numbers of nodal diameters. ${ }^{3}$ This, in essence, provides a tool for obtaining continuous plots of the eigenfrequency versus the number of nodal diameters. Two significant features 
in this type of plot are the slopes of the eigenfrequency curves, and the veerings of these curves that take place at certain frequencies and nodal diameters. These properties are important because they correlate directly with mistuning sensitivity. For example, previous work has shown that curve veerings, in any disordered periodic system, have implications on mode localization. ${ }^{13}$ This, in turn, implies that mistuning sensitivity could be related to the characteristics of veerings and to how close the operating point is to a veering in the eigenfrequency plot.

In addition to correlating with mistuning sensitivity, the free vibration characteristics of a tuned bladed disk can also be related to some important physical notions such as structural interblade coupling and disk/blade modal participation. The structural interblade coupling is governed by the stiffness properties of the disk-blade sector and its connectivity to adjacent sectors. In a structural model of an unshrouded bladed disk, it is conceivable that the dominant means of energy transmission from one blade to the next is through the disk. It is therefore reasonable to assume that the structural interblade coupling is related to the amount of interaction between disk- and blade-dominated modes. Hence, one can assume that the variation of the degree of coupling from one state of operation to another depends on the ratio of disk to blade participation. A direct indication of the relative level of disk participation is the slope of the eigenfrequency locus at the point of operation. The relative level of disk participation can also be related to the characteristics of curve veerings. On this basis, there is a correlation between tuned free vibration characteristics and the physical notions mentioned.

Following from the above discussion, a third link could be established to close a three-way loop. The third connection is between the physical variables (disk/blade participation and structural interblade coupling) on the one hand, and mistuning sensitivity on the other. Indeed, it is well known that for a mistuned bladed disk the degree of structural interblade coupling has a direct effect on mode localization. ${ }^{15}$ To summarize, there is a three-way link among (1) the properties of the tuned structure eigenfrequency curves, (2) physical notions such as disk/blade participation and structural interblade coupling, and (3) the sensitivity of the blades' response to mistuning.

Other factors that come to play in this three-way link are the modal density and the degree of mistuning. It has been reported that for weakly coupled structures mode localization is proportional to the modal density. ${ }^{15}$ However, it was established by the same authors that this is only a necessary, but insufficient requirement for large increases in the forced response. Indeed it was observed that severe mode localization could take place at regions of high modal density, yet in the absence of veering the forced response amplification can very well be low. On the other hand, with the presence of veering, even at a "far distance" from the point of operation, a noticeable increase in the mistuned response is commonly observed. For example, consider a mode set to which a group of excited mistuned modes belong. If the locus of this set veers away at any point, then these excited modes will experience higher amplifications compared to those of a set in which veering does not take place. Therefore, another important physical argument revolves around the influence curve veerings have on producing excessive vibrations regardless of the level of tuned system structural coupling at the point of excitation. The relationship between mistuning sensitivity and the degree of mistuning, at the various operating conditions, is yet another matter of high significance.

A brief description of the reduced order modeling technique and the bladed disk model used in this work is presented in the next section. The following two sections cover the tuned free vibration characteristics, and the corresponding mistuned forced response, respectively. This is then followed by a detailed presentation of the correlations observed, and a comprehensive discussion. Also included is a general set of graphically presented guidelines that could serve as an inexpensive design tool for early assessments of mistuning sensitivity. Finally, the conclusions are drawn in the last section.

\section{VIBRATION ANALYSIS OF A BLADED DISK}

A reduced order modeling technique originally developed by Castanier et al. ${ }^{1}$ and Kruse and Pierre, ${ }^{2}$ and recently extended by Bladh et al., ${ }^{3}$ is used in this study to obtain the mistuned forced response data. The technique produces reduced order models of turbomachinery rotors directly from their finite element models (FEMs) in a systematic fashion. The process involves a component mode analysis of the rotor, with a truncated number of modal amplitudes describing the response of the assembly. Furthermore, the method utilizes cyclic symmetry. Thus only a single sector (Figure 1b) of the bladed disk is modeled rather than modeling the entire assembly (Figure 1a). Blade mistuning is introduced to the FEM by varying the Young's modulus, $E$, in the blade elements by a random variable $\delta$ :

$$
E_{n}=\left(1+\delta_{n}\right) E_{o}, \quad n=1, \ldots, N
$$

where $n$ is the blade number and $N$ is the total number of blades in the assembly. To account for the random nature of blade mistuning, statistical analyses that use Weibull distribution approximations are embedded in the technique. The principal advantage of the reduced 
order modeling method is the considerable computational savings associated with solving for the dynamic response of an entire mistuned bladed disk with a reduced set of degrees of freedom. The research code that implements the above technique is called REDUCE. The reader is referred to Bladh et $a l .^{3}$ for the full mathematical formulations.

The bladed disk analyzed is an industrial 29-blade rotor (Figure 1) which forms the second stage of a four-drum compressor rotor used in an advanced gas turbine application. In this design, the blades and disk are machined from a single, continuous piece of material. Such a design is referred to as a blisk. The material properties for the model are listed in Table 1. The single sector model in Figure $1 \mathrm{~b}$ represents the tuned finite element model, with cyclic symmetry constraints imposed on the disk sector-to-sector boundaries. The model is clamped at the outward rims, which is a rough approximation of boundary conditions due to adjacent rotor stages. The sector finite element model is constructed with linear brick elements (eight-noded solids). The disk portion of the model contains 528 elements, and the blade portion has 375 elements. There are 4374 independent degrees of freedom in the single-sector finite element model.

\begin{tabular}{ll}
\hline Material Property & Property Value \\
\hline Modulus of Elasticity, $E_{0}$ & $203.4 \mathrm{GPa}$ \\
Mass Density, $\rho$ & $7909 \mathrm{~kg} / \mathrm{m}^{3}$ \\
Poisson's Ratio, $v$ & 0.305 \\
Structural Damping, $\gamma$ & 0.006 \\
\hline
\end{tabular}

Table 1. Blisk material properties

\section{TUNED FREE VIBRATION CHARACTERISTICS AND RELATION TO DISK PARTICIPATION OR INTERBLADE COUPLING}

The free vibration characteristics of a tuned bladed disk are best presented through a diagram of natural frequencies versus number of nodal diameters. Figure 2 shows this diagram for the bladed disk under investigation. Note that the method of Bladh et al. ${ }^{3}$, which was referred to in Section 1, is used to obtain continuous plots of the eigenfrequency versus the number of nodal diameters. The diagram exhibits two interesting features that are common for most tuned rotor models. First, the disk stiffens rapidly as the number of nodal diameters increases due to increased disk waviness. Therefore, modes on highly slanted lines in Figure 2 correspond to disk-dominated modes. In contrast, modes on lines that are approximately horizontal correspond to families of blade-dominated modes. Modes on lines that are slightly slanted are "mixed" modes, i.e., modes with both disk and blade contributions. It is hypothesized that without much disk motion, the blades vibrate in isolation. Thus, low and high relative disk participation is associated with weak and strong structural interblade coupling, respectively. This description explains the relation (referred to in Section 1) between the slope of the eigenfrequency locus on the one hand and the relative level of disk participation, and hence structural interblade coupling, on the other. A description of the character of each family of blade-dominated modes is given and denoted by a short code on the far right side of Figure 2.

A second notable feature in Figure 2 is the numerous eigenfrequency veerings ${ }^{16}$ referred to in Section 1. These involve blade- and disk-dominated mode families that veer away from each other. Attention is focused on five distinct eigenfrequency veerings labeled $P$ through $T$. The mode sets (eigenfrequency loci) involved in these veerings are labeled A through $\mathrm{H}$.

An important characteristic of a veering is its sharpness. The sharpness of each of the veerings is purely a tuned system characteristic. The modes in a veering region feature a mix of disk and blade vibration, e.g., $1^{\text {st }}$ disk mode motion and $2 \mathrm{~F}$ blade mode motion at veering P. Hence it can be deduced that the sharpness of a veering depends on the degree of "compatibility" between the two mode types involved. In fact, it is observed that most of the veerings incorporating the $1^{\text {st }}$ disk mode are relatively sharp compared to those involving the $2^{\text {nd }}$ disk mode $\left(2^{\text {nd }}\right.$ highly slanted line to the left). If an operating point coincides with a veering region, the degree of veering sharpness could be related to disk participation, and, in turn, structural interblade coupling as explained above. In a sharp veering mode transformation takes place swiftly (with respect to a change in interblade phase angle), and within the veering there is a small region where the eigenfrequency line is slanted. In contrast, in a smooth veering, mode transformation takes place gradually, and within the veering there is a large region where the eigenfrequency line is slanted (i.e., a large region where the relative level of disk participation is significant). Recalling the previously mentioned relationship between disk participation and structural interblade coupling, it can be concluded that sharp veerings are associated with weak coupling; and that smooth veerings are associated with stronger coupling.

In this study, the tuned free vibration characteristics are defined by the following three distinct variables:

1. The slope of the eigenfrequency locus. Slope steepness is a measure of relative disk participation.

2. The curvature of the eigenfrequency locus. The maximum curvature at a veering is used as an indication of veering sharpness. 
3. The minimum eigenfrequency separation, in $\mathrm{kHz}$, between mode sets at the veering.

Figure 3 graphically demonstrates the definition of each of these tuned free vibration characteristics. Figures 4 through 13 show the veering curvatures and the minimum eigenfrequency locus separations corresponding to Veerings $\mathrm{P}$ through $\mathrm{T}$. The curvatures are computed using a standard fourth-order finite difference scheme. ${ }^{3}$

\section{MISTUNED SYSTEM FORCED RESPONSE AND RELATION TO DISK PARTICIPATION OR INTERBLADE COUPLING}

In this section, the forced response of the mistuned system is considered at a wide range of operating points. At the overall assembly level, a bladed disk can be excited in a manner such that a certain number of nodal diameters appear in the response configuration. This is referred to as engine order excitation (EOE). As mentioned earlier, the blisk under investigation has 29 blades. Therefore, the response under values of EOE ranging from 0 to 14 (assembly's range of harmonics) is computed in order to examine the role of "distance from veering" versus mistuning sensitivity. At the blade level, a single harmonic point load is applied at the tip and mid-chord of each blade, normal to the local surface of the blade. All forced response data presented in this section are obtained utilizing the first five disk modes and the first ten blade modes. Furthermore, the statistical analyses are based on a total of 50 realizations, in which a different mistuning pattern is used for each realization. The degree of mistuning is expressed as a percentage value.

It should be noticed that the eigenfrequency versus number of nodal diameters representation ceases to be valid once mistuning is introduced to the system. The mode pairs of a particular mode family spread in the frequency domain as a result of mistuning, and, unless the degree of mistuning is very weak, nodal lines across the disk no longer become distinctly apparent. An EOE of any value will inevitably excite the entire range of modes that lie within the frequency band of excitation. Naturally, some modes will receive more of the excitation energy than others depending on the location of the operating point. Furthermore, mistuned modes are often perturbations about tuned nodal diameter modes, especially for disk-dominated or mixed modes for which the interblade coupling is relatively strong. It is assumed that the closer the EOE is to the original "tuned" location of the excited mistuned mode, the more this mode will get excited.

The mistuned forced response at frequency ranges in which the blade mode contribution arises from the $2 \mathrm{~F}$ ( $2^{\text {nd }}$ blade flexural mode), $2 \mathrm{~T} / 2 \mathrm{~F}$ (combination of $2^{\text {nd }}$ blade flexural and $2^{\text {nd }}$ blade torsion modes), $2 \mathrm{~T}$ and $3 \mathrm{~F}$ mode families is considered. The variation of the maximum mistuned forced response (among all 29 blades) versus degree of mistuning for the different values of EOE is presented in Figures 14 through 17, respectively, for each of these four mode families. In these figures, the $99.9^{\text {th }}$ percentile values of the statistical response distributions are plotted. Note that the mistuned response is normalized with respect to the response of the tuned state, hence the term, "mistuned response amplification". The variation of the highest value (among all mistuning cases) of the maximum response amplification is shown versus EOE for the same families in Figure 18.

Consider an arbitrary curve among the mistuned response amplification versus percentage mistuning curves presented in Figures 14 through 17. Note that with the introduction of a small amount of mistuning, the response rapidly increases in magnitude. This, as explained in Section 1, is attributed to the fact that any level of disorder in a periodic structure leads to inhibiting the even distribution of vibration energy. This in turn leads to significant vibrations at certain blades due to localization. Interestingly, however, the mistuned response amplification reaches a peak at a certain value of mistuning, then reduces in magnitude and levels off at high values of mistuning. The reason behind the reduction, and subsequent asymptotic leveling, relates to the fact that the participation factors of the mistuned modes become more evenly distributed as mistuning increases. This results in reducing the value of the EOE modal force, hence a reduction in the maximum mistuned response.

The intermediate degree of mistuning with which the maximum forced response is associated will be denoted critical mistuning. Consider the slope of the response amplification curve versus percentage mistuning which is linear at low values of mistuning. It is observed that in most cases when the slope is steep, the maximum response at the critical mistuning value is low, and vice versa. The response amplification slope at low mistuning is therefore a good indication of mistuning sensitivity at critical mistuning. Let this slope be denoted as the amplification-mistuning slope. Figure 19 shows the amplification-mistuning slopes for the four mode families considered.

As mentioned in Section 1, a structure's sensitivity to mistuning correlates with the degree of relative disk participation, and hence structural interblade coupling, at a given point of operation. This concept has been discussed extensively in previous work. ${ }^{3}$ The central idea is that for low coupling, blades vibrate in isolation and there is a reduced probability for localization; whereas for high coupling, each blade's vibration is too sensitive to the motion of adjacent blades and this in turn reduces the probability of energy confinement. 
Consequently, there is a critical value of structural interblade coupling in which mistuning sensitivity is maximized. Associated with this value is a "delicate" level of relative disk participation.

\section{CORRELATIONS BETWEEN TUNED FREE VIBRATION CHARACTERISTICS AND MISTUNING SENSITIVITY (MS)}

\section{MS of a Non-veering Mode Set}

It is known that for weakly coupled structures mode localization is proportional to the modal density. ${ }^{15}$ Based on previous discussion, blade-dominated modes are generally associated with weak structural interblade coupling. Furthermore, it is known that any level of disorder, i.e. mistuning, causes a repeated mode to divide into a multiple set of close modes. The higher the mistuning is, the more scattered the frequencies of the modes are, which in turn means lower modal density. It is therefore reasonable to assume that severe mode localization is likely to occur when a bladedominated mode family of a moderately mistuned system is excited. As an example, consider the $1 \mathrm{~F}$ blade mode family shown in Figure 2. The behavior of this mode family was examined in Bladh et al. ${ }^{17}$ for a rotor very similar to the one analyzed in this study. It was observed that indeed there was severe mode localization when typical densely located mistuned modes of this family were excited. Interestingly, however, it was shown that the set experienced only moderate $(\sim 5 \%)$ amplitude magni-fications. It was therefore established that high modal density in a weakly coupled system is only a necessary, but insufficient requirement for large increases in the forced response. It is of interest to note that the locus of this $1 \mathrm{~F}$ mode set does not feature a veering. Let a set of this type be referred to as a non-veering mode set.

\section{MS Versus Slope of Eigenfrequency Line}

In addition to the level of modal density, another factor that influences the mistuned response is the structural interblade coupling, as demonstrated in Óttarsson and Pierre. ${ }^{15}$ As mentioned earlier, the level of interblade coupling depends on both the structural characteristics and the operating point. If the excited modes belong to a set that has a nearly horizontal eigenfrequency locus, then the relative level of disk participation is low, hence the coupling is weak. On the other extreme, disk dominated modes (i.e. highly slanted eigenfrequency lines) correspond to high structural interblade coupling. In between these two extremes, there are mixed modes, which are associated with intermediate levels of coupling.
Table 2 lists the values of maximum mistuned forced response amplifications for three different disk dominated modes that belong to the $1^{\text {st }}$ disk mode set (see Figure 2). Figure 20 shows the variation of mistuning sensitivity with engine order excitation for the mixed 4F/1R mode family (see Figure 2). This family is chosen because the slope of its eigenfrequency locus, away from the veering, indicates mixed diskblade participation. These two sets of results are obtained utilizing 5 disk modes and 14 blade modes in the reduced order model.

\begin{tabular}{ll}
\hline Disk Mode & Amplification \\
\hline Mode set B, ND 1 & 1.2019 \\
Mode set C, ND 2 & 1.3604 \\
Mode set F, ND 4 & 1.4714 \\
\hline
\end{tabular}

Table 2. Maximum mistuned forced response amplifications for several disk-dominated modes. ND refers to "nodal diameter".

Note that the cases focused on here are ones where the modes in question are located "far" away from any veerings in the eigenfrequency plot. It should also be noted that, unlike the cases of the previous subsection, all these modes belong to sets that have veerings. It is observed that the amplifications $(\sim 10 \%$ to $\sim 50 \%)$ of all modes considered in Table 2 and Figure 20 are moderate, yet higher than those of the non-veering set modes examined in the previous subsection. A possible explanation for this increase refers to the relationship between curve veerings and MS. There are repeated observations in the literature that affirm that modes close to veering regions are highly sensitive to mistuning. Whenever any mistuned mode pair of a veering set gets excited, sensitive modes that are originally close to the veering (i.e., in the tuned case) get excited as well. This contributes to the increase in the response. The importance of how "close" excited modes are from a veering will be elaborated upon in the next sub-section.

It is noticed, from Figure 20, that there is an intermediate region (where the eigenfrequency locus slope is intermediate) of relative disk participation where the mistuned forced response peaks. Specifically, this region corresponds to $\mathrm{EOE}=8$. This verifies results presented in the literature since, as mentioned earlier, the relative level of disk participation is directly proportional to interblade structural coupling. In other words, this is consistent with the hypothesis that the maximum MS is associated with a "critical" value of intermediate structural interblade coupling. Let this hypothesis be referred to as the critical coupling hypothesis.

Another interesting observation regarding modes that are "far" away from veerings is the slight jump in 
amplification that appears at high values of EOE (clearly shown in Figures 18 through 21). This may be attributed to the fact that, regardless of the slope of the eigen-frequency line, structural interblade coupling is also related to the number of nodal diameters. As the number of nodal diameters increases, the disk becomes stiffer. This results in a lower level of interblade coupling since the avenue of vibration transmission via the disk is reduced. Based on this description, a response peak (these jumps at high EOE) is associated with an intermediate level of structural interblade coupling. Again, this validates the critical coupling hypothesis.

\section{MS Versus Distance from Veering}

It is clear from the mistuned forced response diagrams (Figures 14 through 18) that the highest values of maximum amplification occur in regions close to veerings. It could be assumed that, at the exact center of a veering, modes are almost equally mixed in disk and blade participation. Since diskdominated modes feature strong coupling and bladedominated modes feature weak coupling, it is likely that the critical (intermediate) level of coupling occurs near a veering.

To check the validity of the critical coupling hypothesis in this context (i.e., effect of the "distance from veering"), a new set of similar bladed disk models are constructed with varying disk properties. The purpose of this is to create cases where the curve veering under examination is located at different locations across the number of nodal diameters axis. The variation of the disk stiffness leads to a change in the location of the veering from the value of applied engine order excitation. This is an indirect way of examining the influence the "distance from veering" has on MS. Figure 21 shows the eigenfrequency loci for values of disk stiffness varying from $90 \%$ to $110 \%$ of some nominal value. Note the shifting of the "center" of the curve veering. A stiffness value of $\mathrm{E} \approx 91 \%$ corresponds to the case where the $2^{\text {nd }}$ nodal diameter (value of the applied EOE) lies exactly at the "center" of the veering. Figure 22 shows the relationship between MS and the value of disk flexibility, which, as mentioned above, reflects the relationship between $\mathrm{MS}$ and the nodal diameter distance between the value of EOE and the veering. Both Figures 21 and 22 are obtained from Bladh et $a l{ }^{18}$ It is noticed that the mistuned forced response amplification has a local minimum at the "center" of the veering. This result shows that at the exact "center" of the veering, the relative level of disk motion is much higher than the level required in the critical case. More importantly, it is observed that a maximum value of MS is associated with a certain nodal diameter distance from the veering, as would have been predicted by the critical coupling hypothesis. The precise level of structural interblade coupling at this distance is the critical level. Associated with this level is a delicate balance of disk and blade participation.

\section{MS Versus Veering Characteristics}

Consider the cases where the EOE is at, or close to, the actual veering region. Here, as shown in Figures 14 through 18, the mistuned response amplification is bound to be most severe. This is because most of the excitation energy (due to the closeness of the value of EOE) works on exciting the modes that are at the veering, which commonly are critically coupled, or at least close to such. In this context, the characteristics of the eigenfrequency veering are expected to clearly correlate with the extent of mistuning sensitivity. The five veerings labeled $\mathrm{P}$ through $\mathrm{T}$ in Figure 2 are considered. Table 3 summarizes the relationship between the characteristics of these veerings and the corresponding amplitudes of the mistuned response amplification.

From Table 3, it is noticed that there is an intermediate level of veering curvature with which maximum MS is associated. Namely, Veering $\mathrm{R}$ has this critical curvature value (4.83). In reference to the critical coupling hypothesis, it can be deduced that the level of structural interblade coupling corres-

\begin{tabular}{|c|c|c|c|c|c|}
\hline Veering & $\mathrm{P}$ & $\mathrm{Q}$ & $\mathrm{R}$ & $\mathrm{S}$ & $\mathrm{T}$ \\
\hline Disk Mode & $1^{\mathrm{st}}$ & $1^{\mathrm{st}}$ & $2^{\text {nd }}$ & $2^{\text {nd }}$ & $3^{\text {rd }}$ \\
\hline Blade Mode & $2 \mathrm{~F}$ & $2 \mathrm{~T} / 2 \mathrm{~F}$ & $2 \mathrm{~T}$ & $3 \mathrm{~F}$ & $3 \mathrm{~F}$ \\
\hline Nodal Diameter & 1 & 2 & 2 & 3 & 2 \\
\hline Max. Curvature & 12.83 & 6.70 & 4.83 & 3.31 & 3.28 \\
\hline $\begin{array}{l}\text { Min. Frequency } \\
\text { Separation }[\mathrm{kHz}]\end{array}$ & 0.35 & 0.80 & 1.35 & 1.05 & 0.74 \\
\hline Max. Amplif. & 2.14 & 2.71 & 2.86 & 2.79 & 2.67 \\
\hline $\begin{array}{l}\text { Mistuning [\%] for } \\
\text { Max. Amplif. }\end{array}$ & 0.8 & 2.5 & 3.0 & 2.5 & 1.7 \\
\hline $\begin{array}{l}\text { Slope of Max. } \\
\text { Amplif. Versus } \\
\text { Mistuning, at } \\
\text { Low Mistuning }\end{array}$ & 557 & 466 & 432 & 376 & 404 \\
\hline
\end{tabular}

Table 3. Tuned vibration characteristics and corresponding mistuning sensitivity information for veerings labeled in Figure 2. The last two rows refer to the critical mistuning and the amplificationmistuning slope, respectively. Note that the highest maximum amplification is associated with veering $R$. 
ponding to the tuned mode pair that lies at the edge of Veering $\mathrm{R}$ is the critical level (in comparison to the levels associated with the other considered veerings). Table 3 also shows the relationship between MS and yet another veering characteristic: the minimum eigenfrequency distance at the veering. The results show that this minimum veering separation distance is highest for Veering $\mathrm{R}$, the veering associated with the critical MS. This observation, if proven to be generally true, would suggest that the minimum veering separation distance is a simple and straightforward indication of MS.

\section{MS Versus Degree of Mistuning}

Table 3 also shows the mistuning level responsible for the maximum MS (i.e. critical mistuning) corresponding to Veerings $\mathrm{P}$ through T. Upon comparing the values of critical mistuning for the veerings, it is noticed that this quantity peaks $(3 \%)$ for the critically coupled case associated with Veering $\mathrm{R}$. This result implies that the closer the structural interblade coupling is to the critical level, the higher the degree of mistuning needed to cause maximum MS. Furthermore it is noticed that the amplificationmistuning slope for Veering $\mathrm{R}$ is relatively low (the lowest slope is that of Veering S). This observation supports, to some extent, the hypothesis that the value of critical mistuning is inversely proportional to the amplification-mistuning slope.

\section{DISCUSSION}

\section{Summary of Factors that Contribute to Mistuning} Sensitivity

The results presented confirm that there is a critical degree of structural interblade coupling at which MS is maximized. Physically it is conceivable, and indeed shown in Figure 20, that associated with the critical coupling level there is a delicate balance of disk and blade participation. Hence, it can generally be viewed that structural interblade coupling and the relative level of disk participation are directly related. In fact, the former can be considered to be a consequence of the latter. It should be noted that the dynamic interblade coupling referred to here is a variable that changes with the state of operation. The level of dynamic interblade coupling depends on the tuned free vibration characteristics, as well as the operating conditions, through the following four factors:

1. Nodal diameter distance between veering and excited mode pair.

2. Curvature of veering near which an excited mode pair lies.
3. Slope of eigenfrequency line to which an excited mode pair belong.

\section{Value of nodal diameter (or EOE).}

Based on the results presented, the above four factors are roughly ordered in descending order of significance with regards to the effect critical coupling has on increasing the MS. At this point, it is worth noting that such order may change from one model to another. The increase in MS is measured in comparison to the value of MS at a non-critically coupled state belonging to the same mode set. It is noticed that the degree of increase in MS associated with the combined effect of the first and second factors ( $\sim 65 \%$ to $\sim 110 \%)$ is commonly much higher than that is associated with the $3^{\text {rd }}$ and 4th factors ( $\sim 25 \%$ and $\sim 10 \%$, respectively). This is apparent from comparing the corresponding increases in mistuned forced response amplification. Data related to the $1^{\text {st }}$, $2^{\text {nd }}$ and $4^{\text {th }}$ factors is read from Figures 14 through 17; and data related to the $3^{\text {rd }}$ factor is read from Figure 20. Note that curve veerings are the prime cause for major increases in MS. Recall that at a veering mode shape transformation takes place.

The significant influence of curve veerings sheds light upon the importance of their detailed characteristics, and upon the relevance of how close a veering is to the EOE. With these two factors considered, as well as the other factors mentioned, there is clearly a substantial degree of complexity associated with the problem of predicting mistuning sensitivity of a bladed disk from tuned free response information. Figure 23 graphically summarizes the three-way link referred to in the first section. Note the presence of the "critical" region associated with the various metrics and quantities presented. Also included in Figure 23 is a brief description of the underlying physical mechanisms that relate MS to structural interblade coupling.

\section{General Guidelines for Bladed Disk Design}

The most ambitious outcome of the analyses and insights presented in this paper would be a set of design guidelines for a mistuning-resistant turbomachinery rotor. A preliminary version of such a set of guidelines is presented in Figure 24, which shows a schematic diagram that classifies the various operating zones, qualitatively, and indicates the corresponding "anticipated" MS. It is important to note that the diagram and guidelines are based on the results and analyses presented in this study, as well as some reported results from the literature, but they will not hold for all cases. It is also worth noting that the conclusions arrived at here are based on an integrated view of all the factors referred to in this study, and that 
the relative importance of these factors may differ from one physical model to another. However, it is believed that this type of approach could eventually be used for the development of an inexpensive design tool for early assessments of mistuning sensitivity.

\section{CONCLUSIONS}

This paper focused on the analyses of the complex dynamic characteristics of an industrial bladed disk. The study explored the three-way connection, and associated relationships, among (1) tuned free vibration characteristics, (2) physical notions such as interblade coupling and disk/blade participation, and (3) mistuning sensitivity of blade vibrations.

The characteristics of the eigenfrequency versus number of nodal diameters plot, supplemented with a recently published interblade phase angle representation, were used as the basis for quantifying the tuned free vibration characteristics. Furthermore, an advanced reduced order modeling technique was used as a tool to obtain a large set of mistuned forced response data. With the tuned free vibration characteristics quantified, and the mistuned response data in hand, several sets of comprehensive correlative analyses were conducted and conclusions were derived.

It was concluded that there are numerous intertwining factors that affect mistuning sensitivity in a mistuned bladed disk. Most important is the structural interblade coupling (also related to the relative level of disk participation) that varies from an operating state to another. Results show that there is a critical value of coupling corresponding to maximum mistuning sensitivity. The influence of both the location and the characteristics of eigenfrequency veerings were proven to be of paramount importance. All the relevant factors were presented in connection to the eigenfrequency versus number of nodal diameters plot for the tuned system. Furthermore, the effects of these factors were presented quantitatively, and were thoroughly discussed from a physical perspective.

Finally, on the basis of the results and physical insights gained, specific operation zones in the eigenfrequency versus nodal diameter plot were classified in terms of "anticipated" safety of operation. This classification is viewed as a general set of guidelines, or an inexpensive design tool, for early assessments of mistuning sensitivity.

\section{ACKNOWLEDGEMENTS}

The authors gratefully acknowledge Dr. Ronnie Bladh for his insights and for providing several of the figures presented in this paper.

\section{REFERENCES}

1. Castanier, M. P., Óttarsson, G., and Pierre, C., 1997, "A reduced-order modeling technique for mistuned bladed disks," Journal of Vibration and Acoustics, Vol. 119(3), pp. 439-447.

2. Kruse, M. J. and Pierre, C., 1996, "Forced response of mistuned bladed disks using reduce-order modeling," Proceedings of the $37^{\text {th }}$ AIAA/ASME Structures, Structural Dynamics, and Materials Conference, Salt Lake City, Utah.

3. Bladh, R., Pierre, C., Castanier, M. P., and Kruse, M. J., 2002, "Dynamic response predictions for a mistuned industrial turbomachinery rotor using reduced order modeling," ASME Journal of Engineering for Gas Turbines and Power, Vol. 124(2), pp. 311-324.

4. Anderson, P. W., 1958, "Absence of diffusion in certain random lattices," Physical Review, Vol. 109, pp. 1492-1505.

5. Montroll, E. W. and Potts, R. B., 1955, "Effects of defects on lattice vibrations," Physical Review, Vol. 100, pp. 525-543.

6. Robenstock, H. B. and McGill, R. E., 1968, "Vibrations of disordered solids," Physical Review, Vol. 176, pp. 1004-1014.

7. Ishii, K., 1973, "Localization of eigenvalues and transport phenomena in the one-dimensional disordered system," Supplement of the Progress of Theoretical Physics, Vol. 53, pp. 77-138.

8. Hodges, C. H., 1982, "Confinement of vibration by structural irregularity," Journal of Sound and Vibrations, Vol. 82(3), pp. 441-424.

9. Valero, N. A. and Bendiksen, O. O., 1986, "Vibration characteristics of mistuned shrouded blade assemblies," ASME Journal of Engineering for Gas Turbines and Power, Vol. 108(2), pp. 293299.

10. Bendiksen, O. O., 1987, "Mode localization phenomena in large space structures," AIAA Journal, Vol. 25(9), pp. 1241-1248.

11. Pierre, C. and Dowell, E. H., 1987, "Localization of vibrations by structural irregularity," Journal of Sound and Vibrations, Vol. 114, pp. 549-564.

12. Pierre, C., Tang, D. M., and Dowell, E. H., 1987, "Localized vibrations of disordered multi-span beams: theory and experiment," AIAA Journal, Vol. 25(9), pp. 1249-1257.

13. Pierre, C., 1988, "Mode localization and eigenvalue loci veering phenomena in disordered structures," Journal of Sound and Vibrations, Vol. 126(3), pp. 485-502.

14. Pierre, C., 1990, "Weak and strong vibration localization in disordered structures: a statistical investigation," Journal of Sound and Vibrations, Vol. 139(1), pp. 111-132. 
15. Óttarsson, G. S. and Pierre, C., 1995, "On the effects of interblade coupling on the statistics of maximum forced response amplitudes in mistuned bladed disks," in Proceedings of the $36^{\text {th }}$ AIAA/ASME Structures, Structural Dynamics, and Materials Conference, New Orleans, Louisiana.

16. Perkins, N. C. and Mote Jr, C. D., 1986, "Comments on curve veering in eigenvalue problems," Journal of Sound and Vibrations, Vol. 106, pp. 451-463.

17. Bladh, R., Castanier, M. P., and Pierre, C., 1998, "Reduced order modeling and efficient forced response statistics prediction for mistuned bladed disks," Proceedings of the $3^{\text {rd }}$ National Turbine Engine High Cycle Fatigue Conference, San Antonio, Texas.

18. Bladh, R., Castanier, M. P., and Pierre, C., 2003, "Effects of Multistage Coupling and Disk Flexibility on Mistuned Bladed Disk Dynamics," ASME Journal of Engineering for Gas Turbine and Power, Vol. 125(1), pp. 121-130.

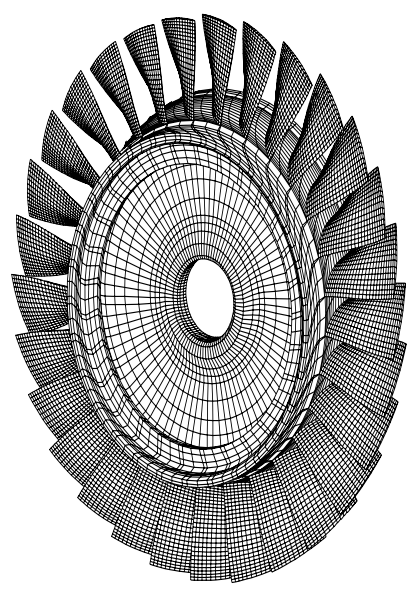

(a)

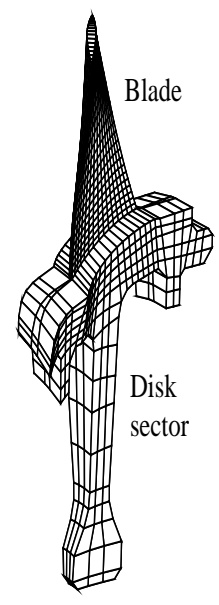

(b)

Figure 1. Finite element meshes for the industrial 29-blade compressor rotor: (a) full assembly; (b) cyclic disk-blade sector model.

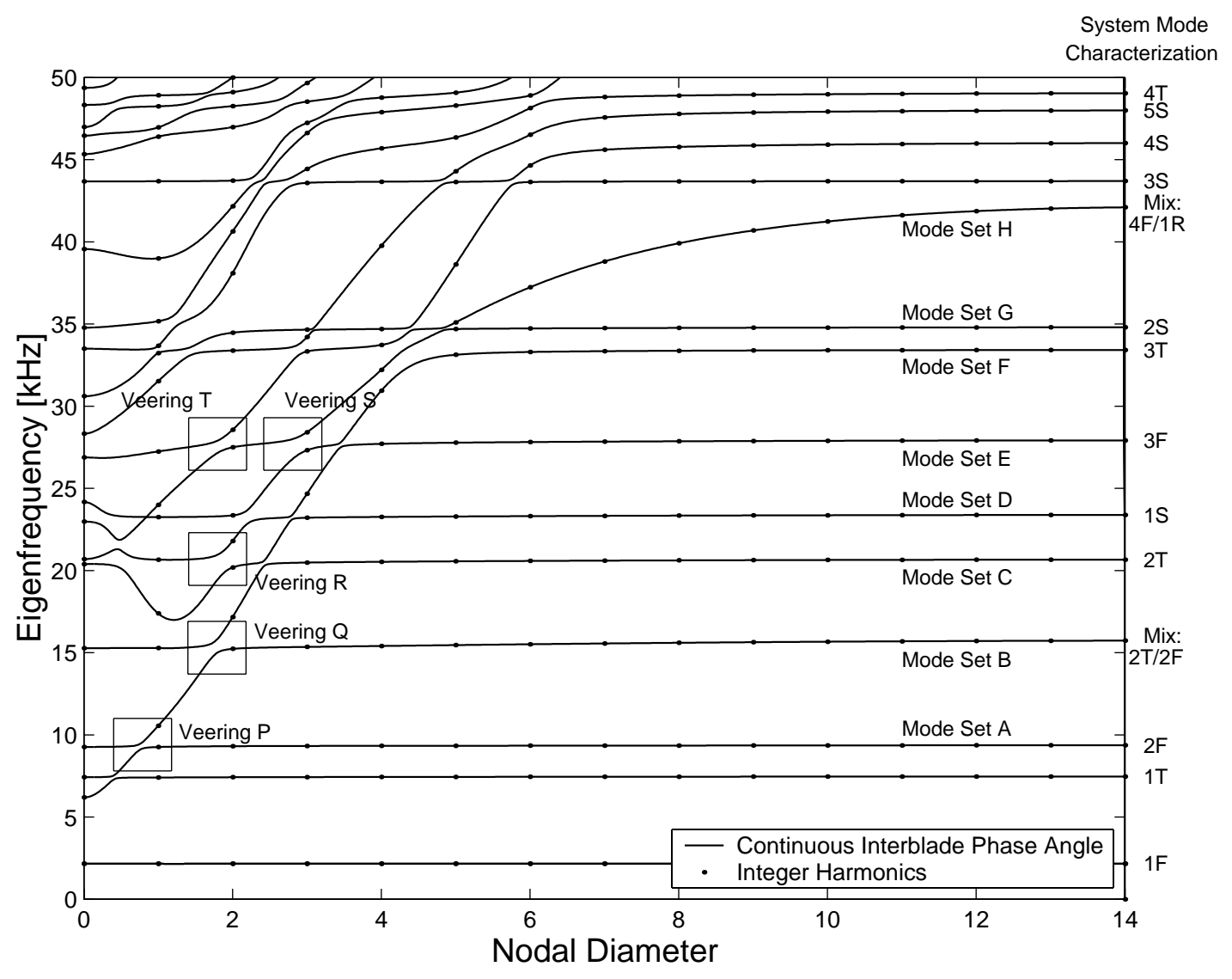

Figure 2. Natural frequencies versus number of nodal diameters for the tuned rotor. The character of each family of blade-dominated modes is indicated on the right, where $\mathrm{F}=\mathrm{Flexural}, \mathrm{T}=\mathrm{T}$ orsion, $\mathrm{S}=$ Stripe, and $\mathrm{R}=$ Radial. 


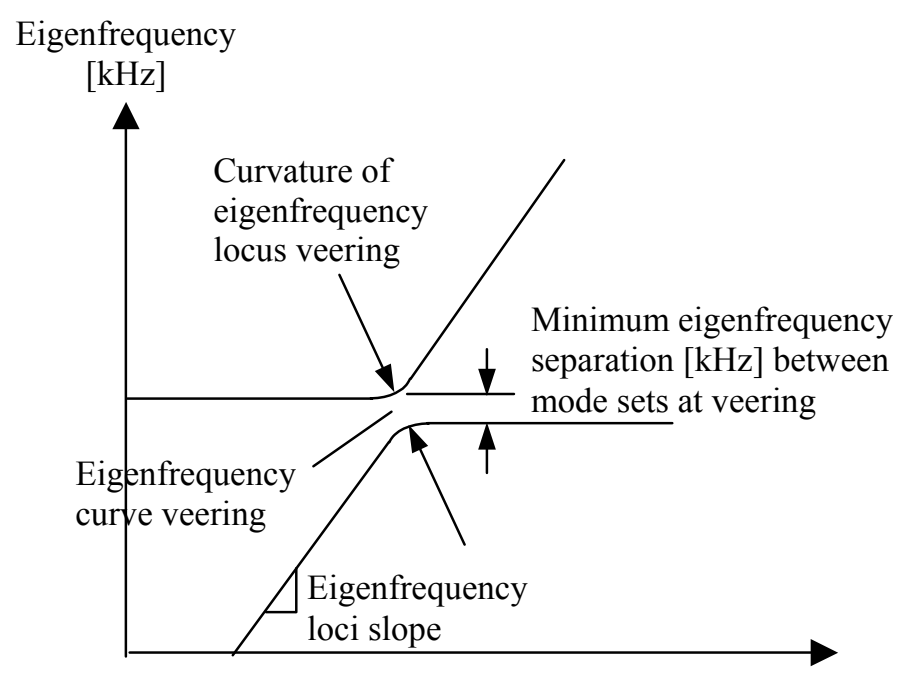

Engine Order Excitation

Figure 3. Geometric characteristics of curve veering.

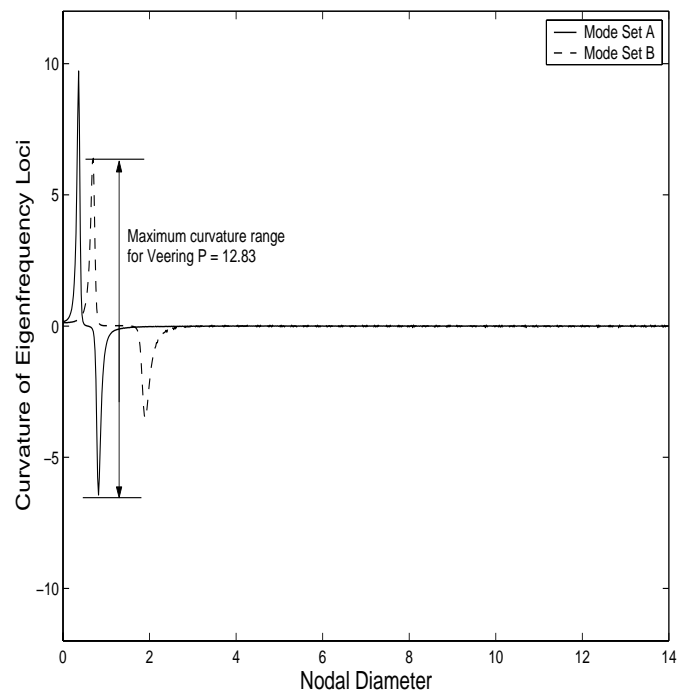

Figure 4. Finite difference curvature of the eigenfrequency locus for mode sets A and B.

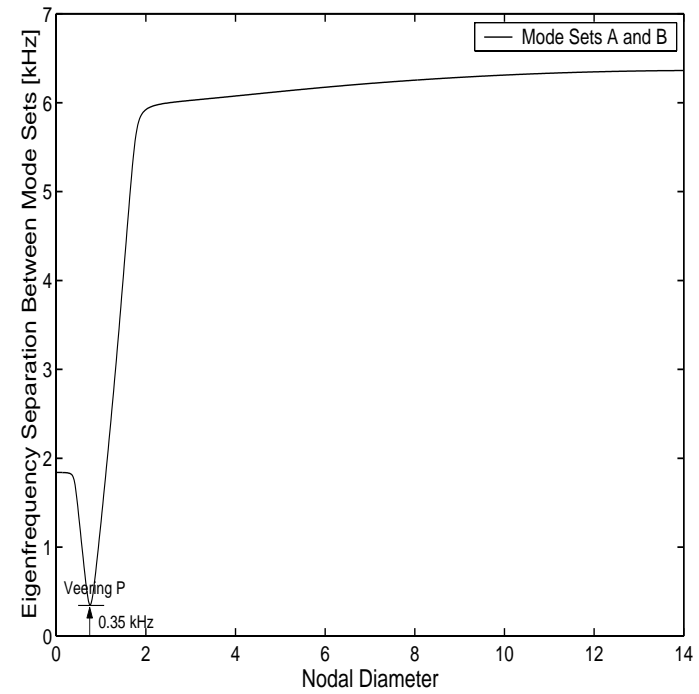

Figure 5. Eigenfrequency separation $[\mathrm{kHz}]$ between the eigenfrequency locus of mode sets A and B. 


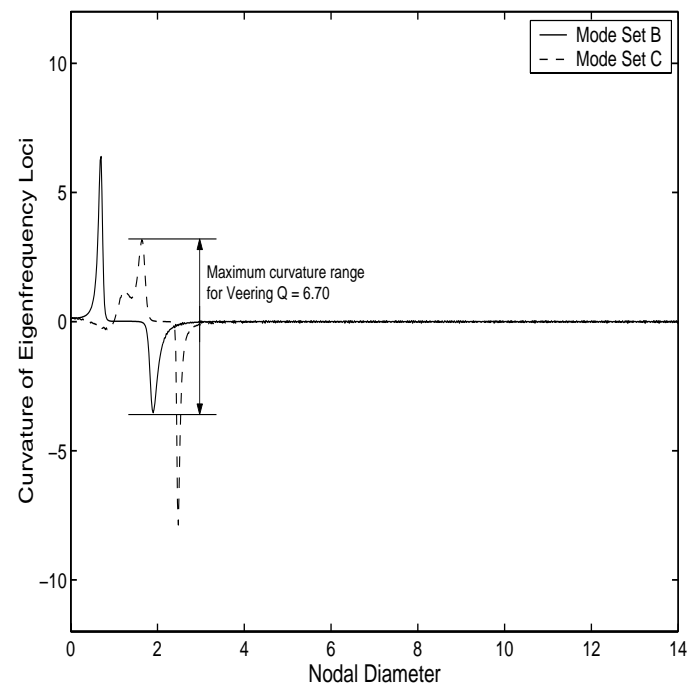

Figure 6. Finite difference curvature of the eigenfrequency locus for mode sets B and C.

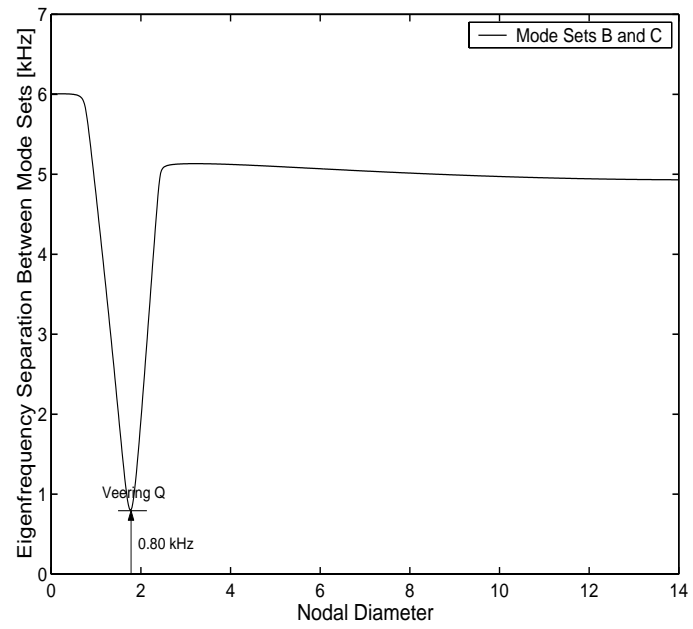

Figure 7. Eigenfrequency separation $[\mathrm{kHz}]$ between the eigenfrequency locus of mode sets B and C.

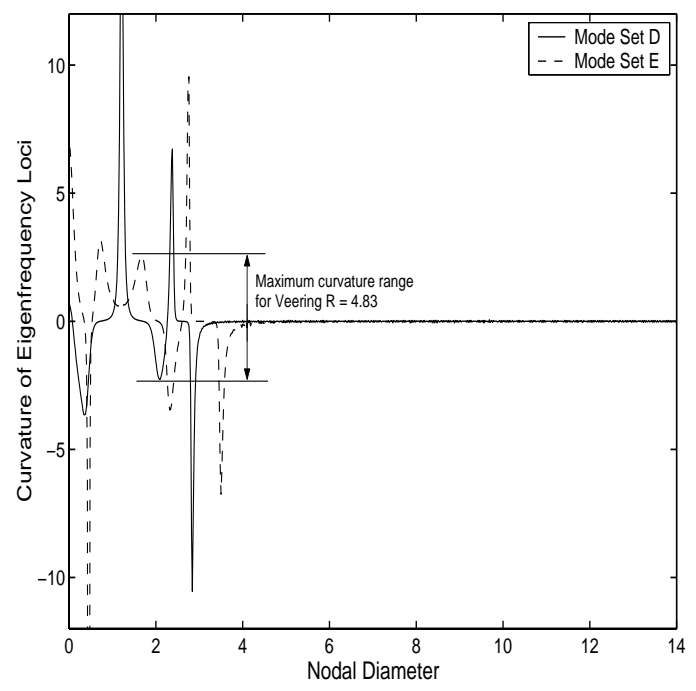

Figure 8. Finite difference curvature of the eigen frequency locus for mode sets D and E.

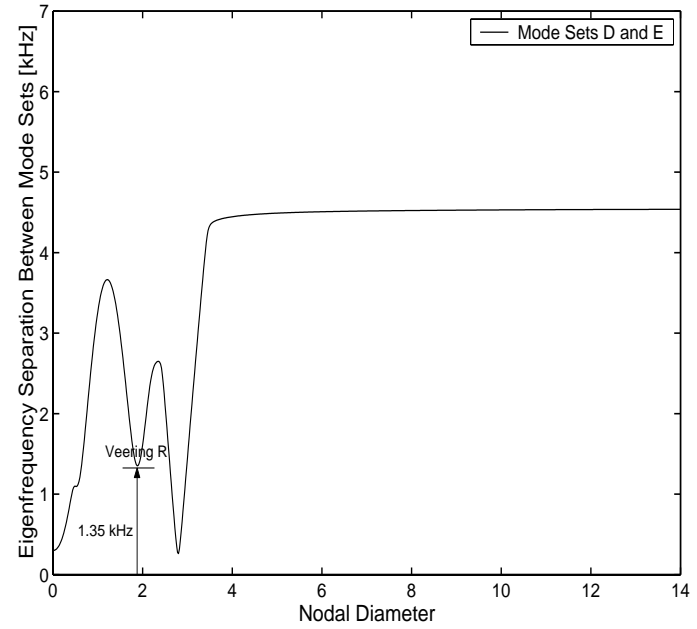

Figure 9. Eigenfrequency separation $[\mathrm{kHz}]$ between eigenfrequency locus of mode sets D and E. 


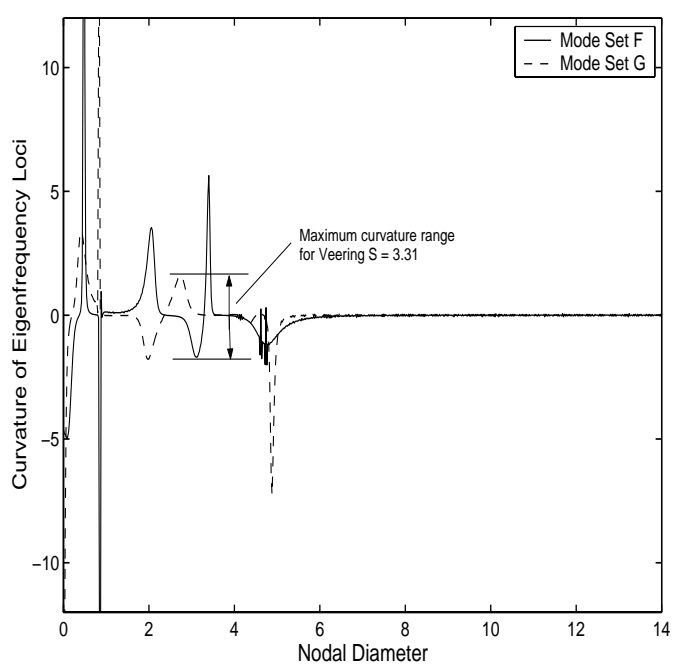

Figure 10. Finite difference curvature of the eigenfrequency locus for mode sets F and G.

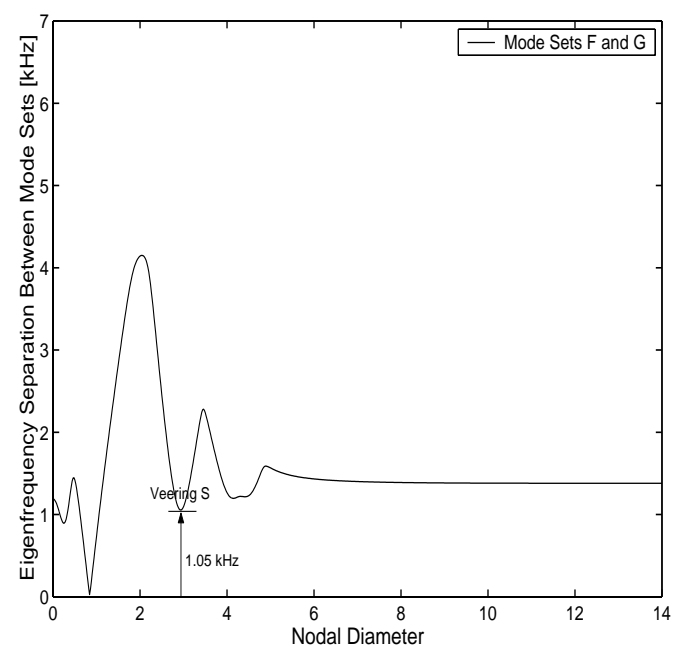

Figure 11. Eigenfrequency separation $\mathrm{kHz}]$ between the eigenfrequency locus of mode sets $\mathrm{F}$ and $\mathrm{G}$.

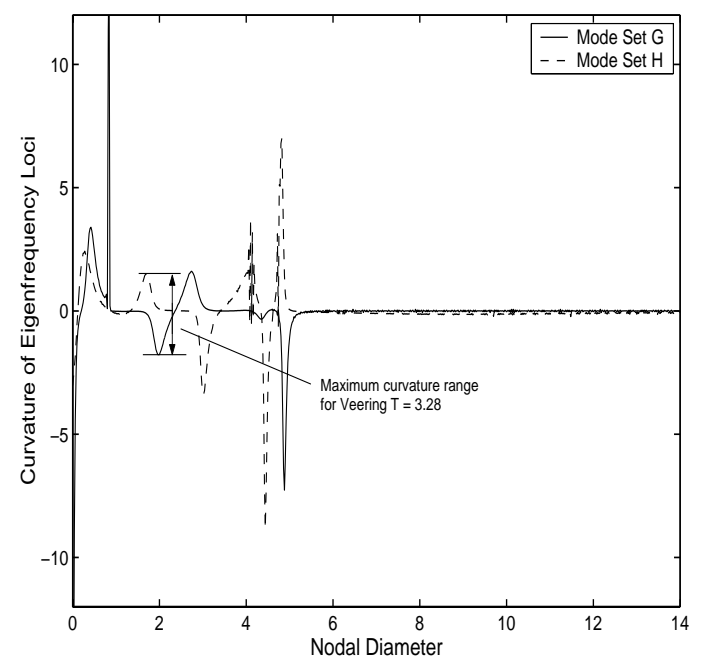

Figure 12. Finite difference curvature of the eigen-frequency locus for mode sets $\mathrm{G}$ and $\mathrm{H}$.

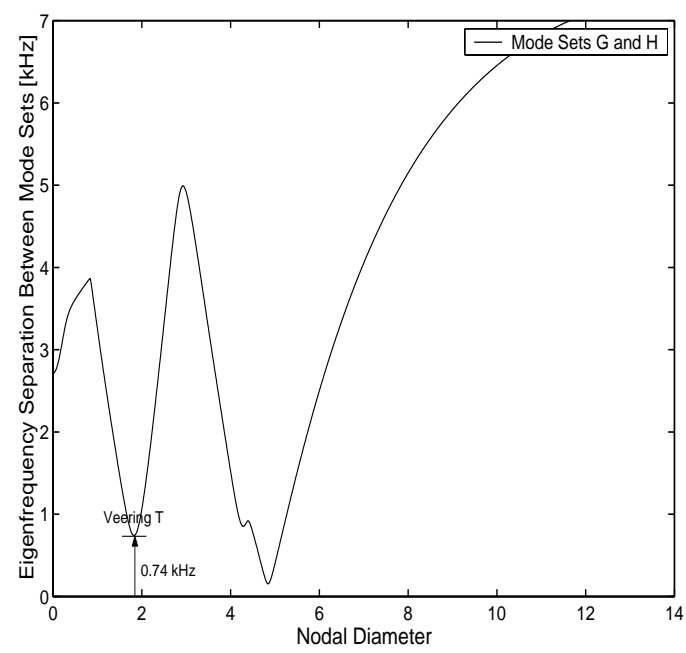

Figure 13. Eigenfrequency separation $[\mathrm{kHz}]$ between the eigenfrequency locus of mode sets $\mathrm{G}$ and $\mathrm{H}$. 

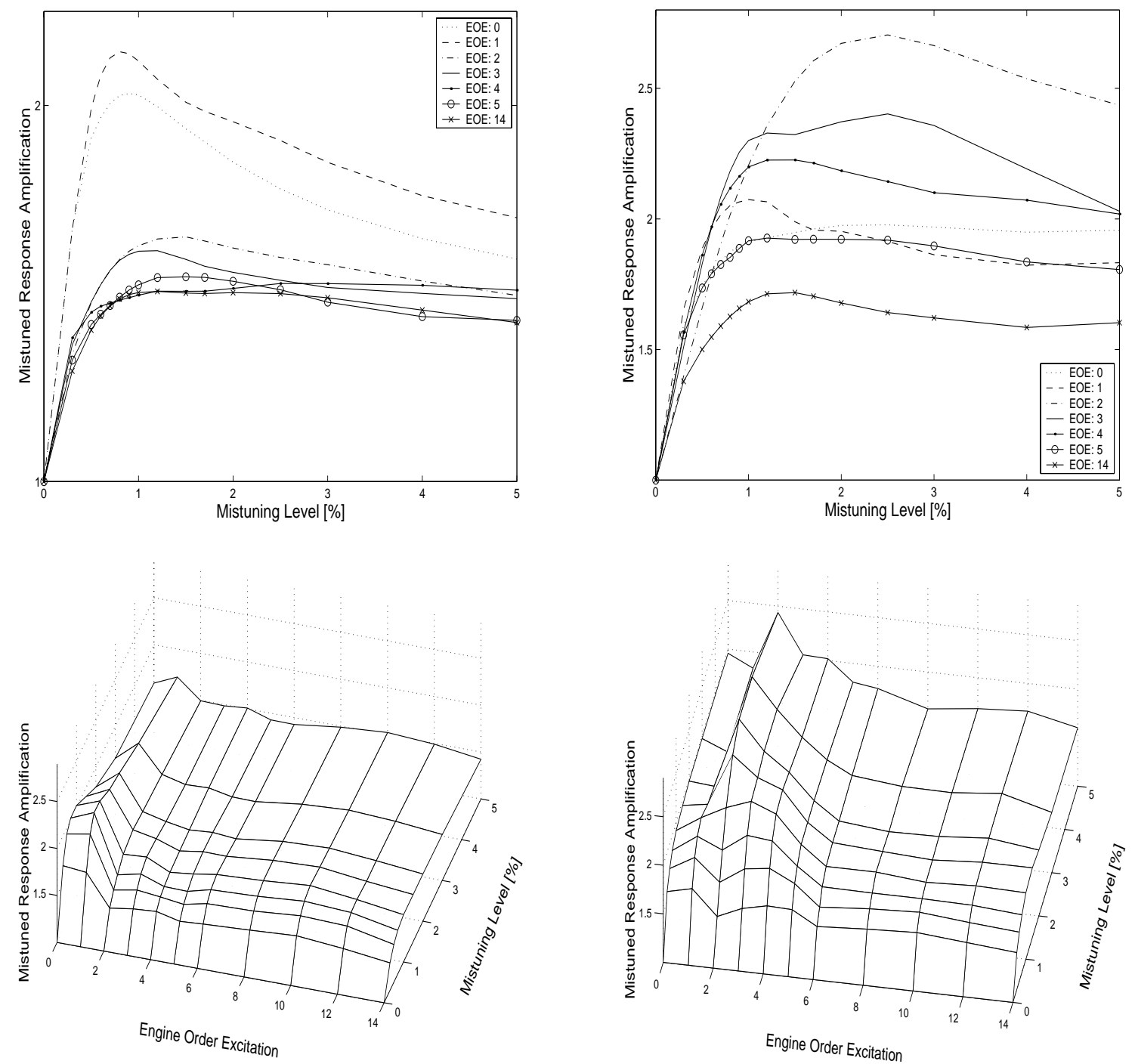

Figure 14. Variation of maximum forced response amplification with the degree of mistuning for the $2 \mathrm{~F}$ mode family for various values of engine order excitation.

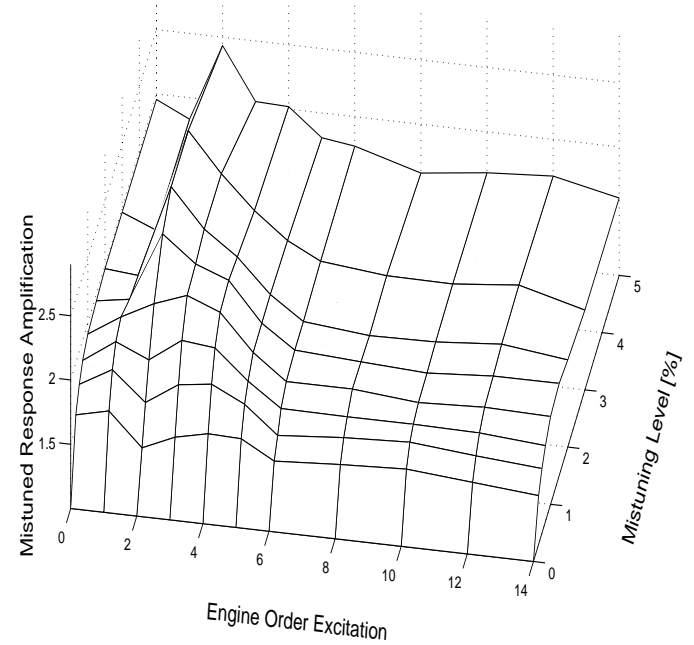

Figure 15. Variation of maximum forced response amplification with the degree of mistuning for the $2 \mathrm{~T} / 2 \mathrm{~F}$ mode family for various values of engine order excitation. 

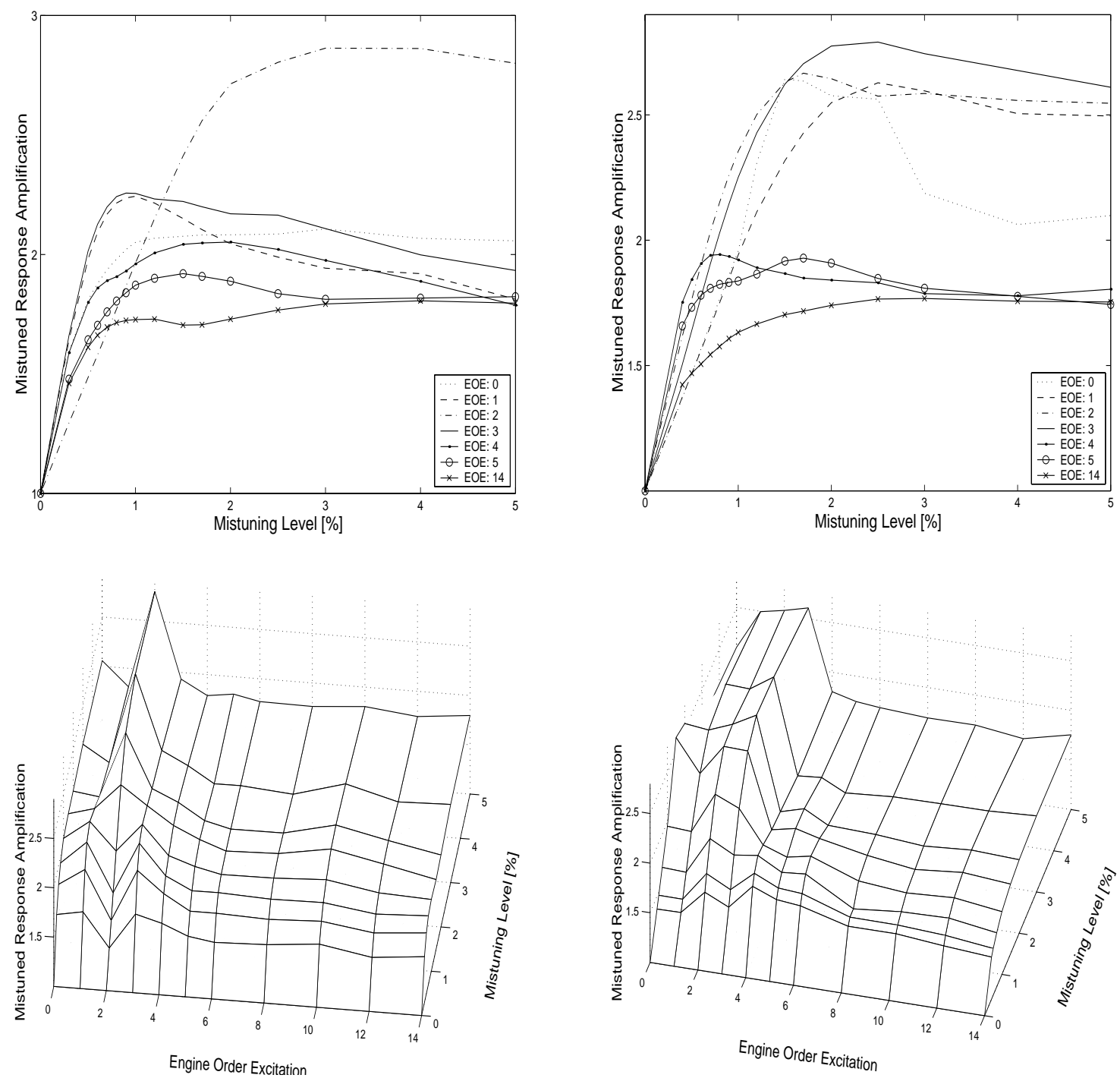

Figure 16. Variation of maximum forced response amplification with the degree of mistuning for the $2 \mathrm{~T}$ mode family for various values of engine order excitation.

Figure 17. Variation of maximum forced response amplification with the degree of mistuning for the $3 \mathrm{~F}$ mode family for various values of engine order excitation. 


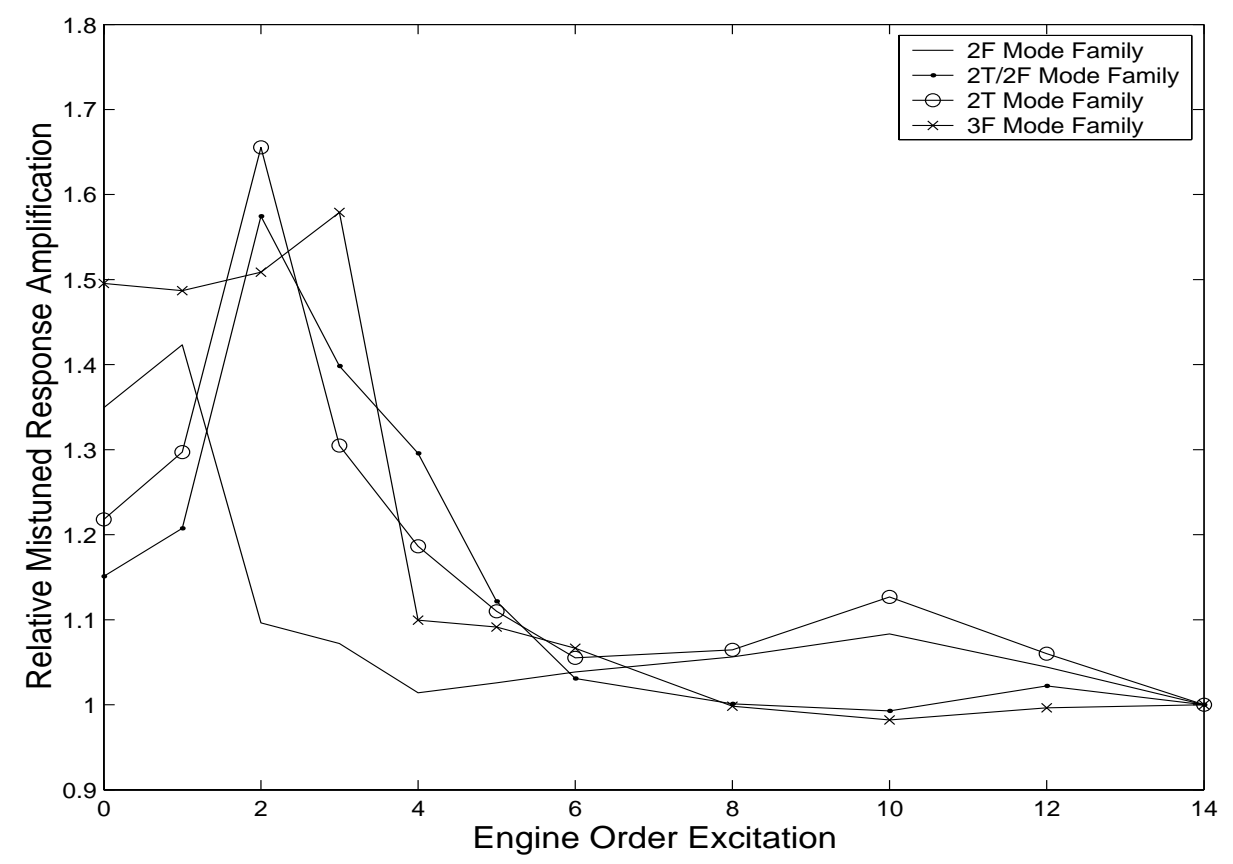

Figure 18. Variation of highest mistuned forced response amplification (among all mistuning cases) with engine order excitation for the $2 \mathrm{~F}, 2 \mathrm{~T} / 2 \mathrm{~F}, 2 \mathrm{~T}$ and $3 \mathrm{~F}$ mode families. The data for each mode family is normalized with respect the amplification value at EOE 14.

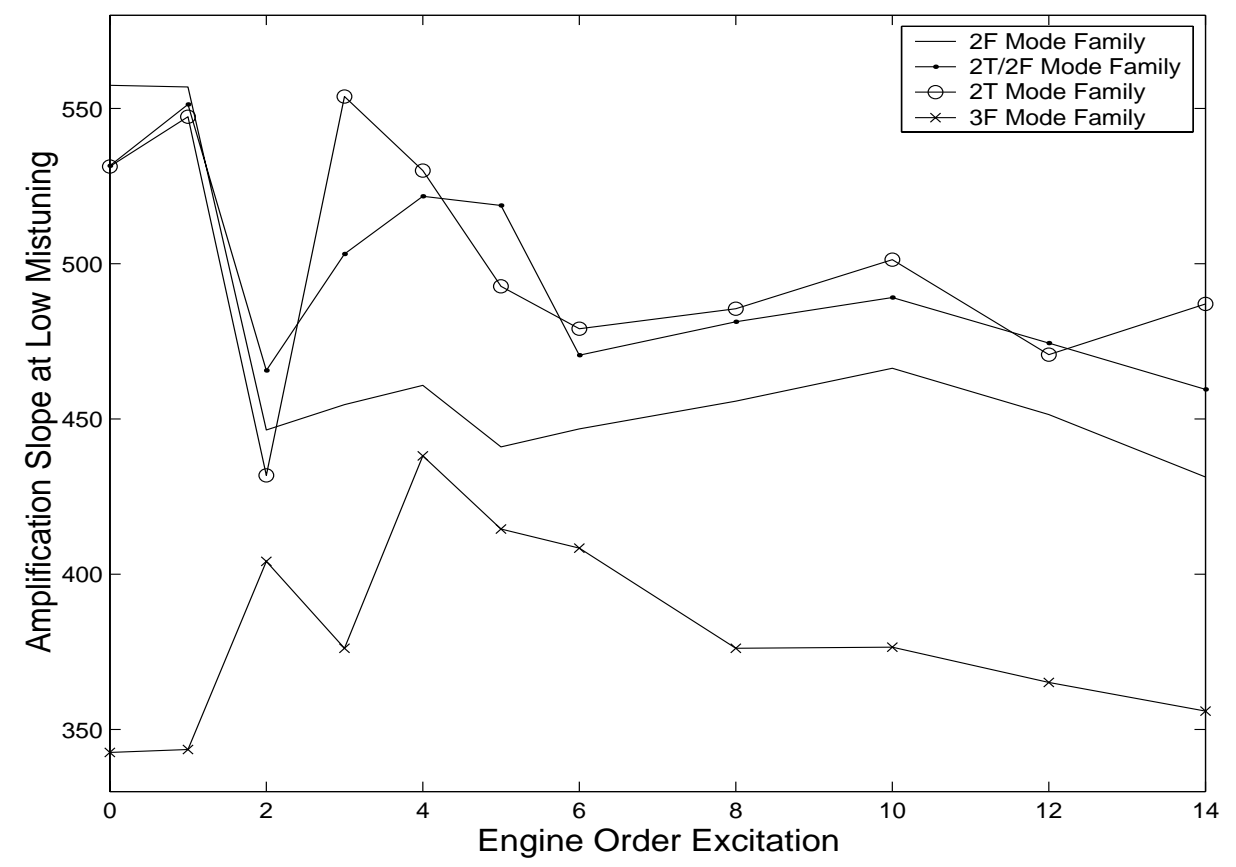

Figure 19. Variation of amplification-mistuning slope at low mistuning with engine order excitation for the $2 \mathrm{~F}, 2 \mathrm{~T} / 2 \mathrm{~F}, 2 \mathrm{~T}$ and $3 \mathrm{~F}$ mode families. 


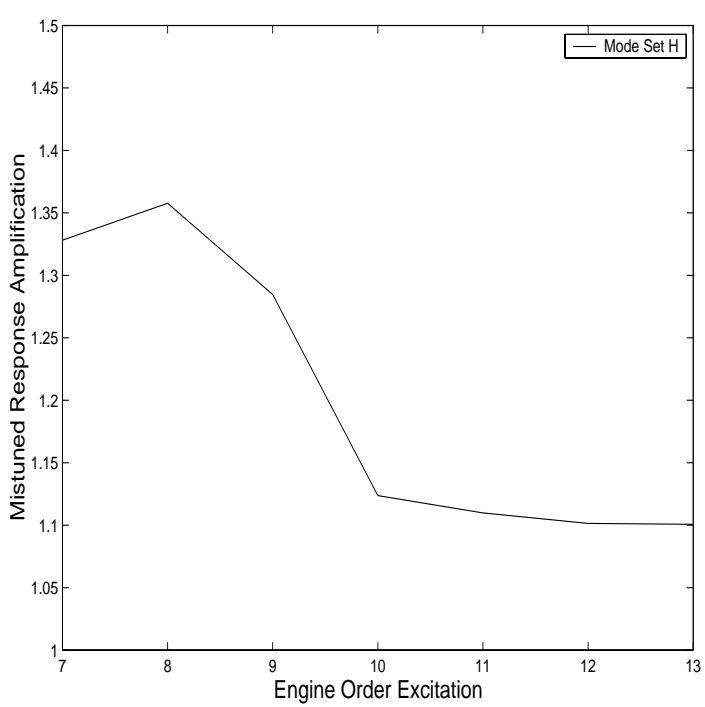

Figure 20. Variation of mistuning sensitivity with engine order excitation for the mixed $4 \mathrm{~F} / 1 \mathrm{R}$ mode set.

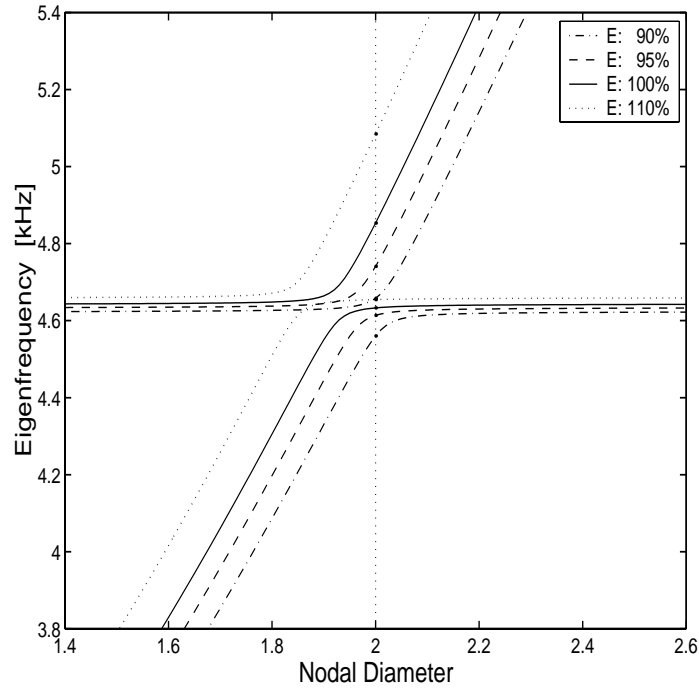

Figure 21. Eigenfrequency loci for different values of disk stiffness ${ }^{18}$. Note the shifting of the "center" of the curve veering.

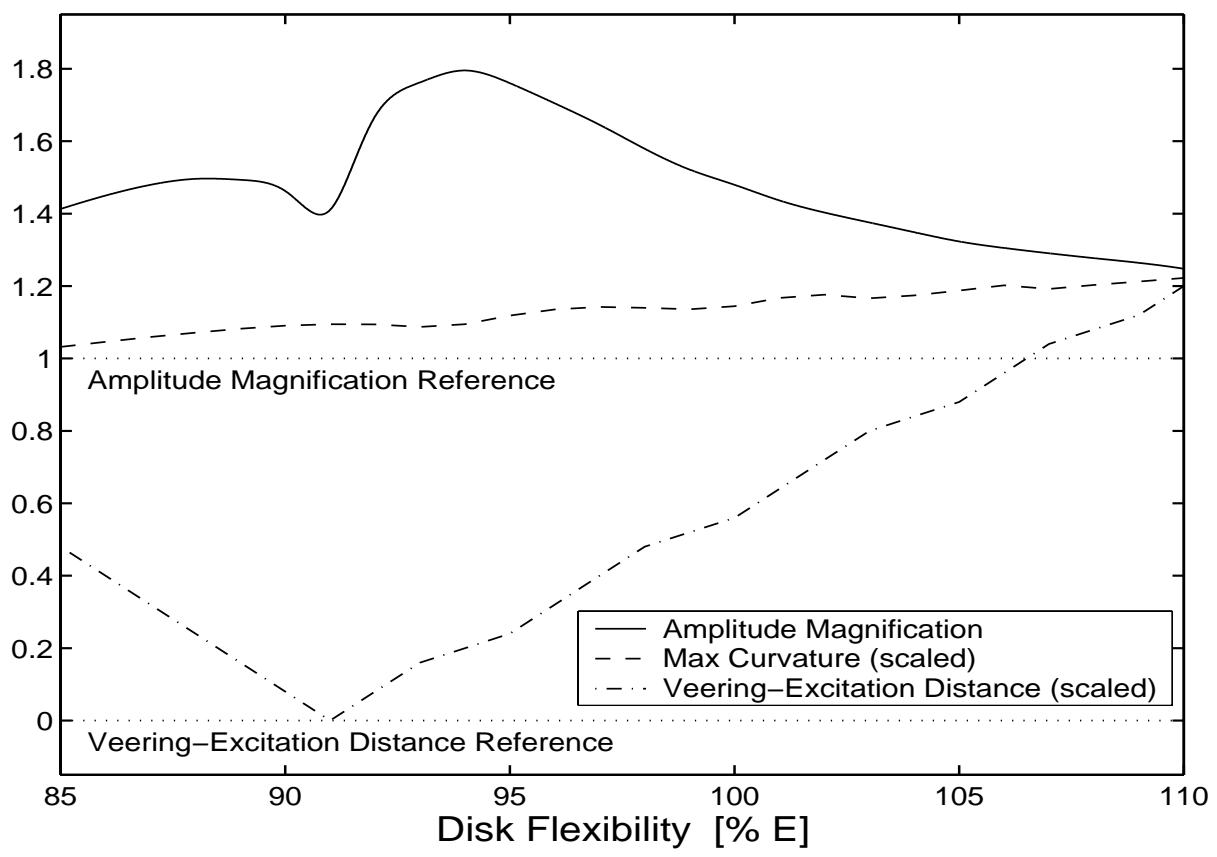

Figure 22. Relationship between mistuning sensitivity and nodal diameter distance from veering. This relationship is produced by varying disk flexibility, hence varying location of veering from the value of applied engine order excitation ${ }^{18}$. 


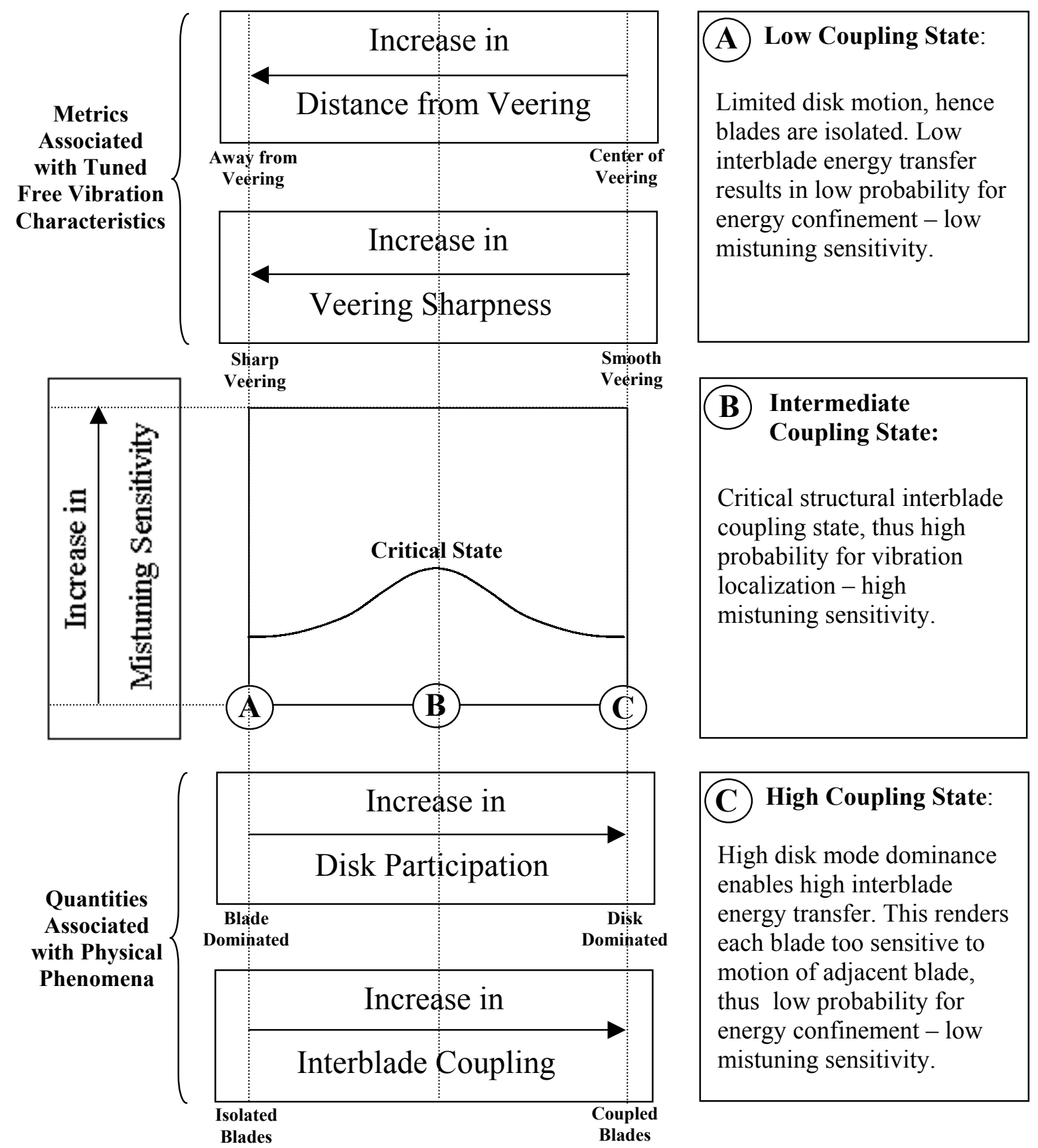

Figure 23. A graphical summary of the three-way link among (1) tuned structure eigenfrequency information, (2) physical notions such as disk participation and structural interblade coupling, and (3) mistuning sensitivity. Also shown is a description of the physical mechanisms responsible for mistuning sensitivity at the various coupling levels. 


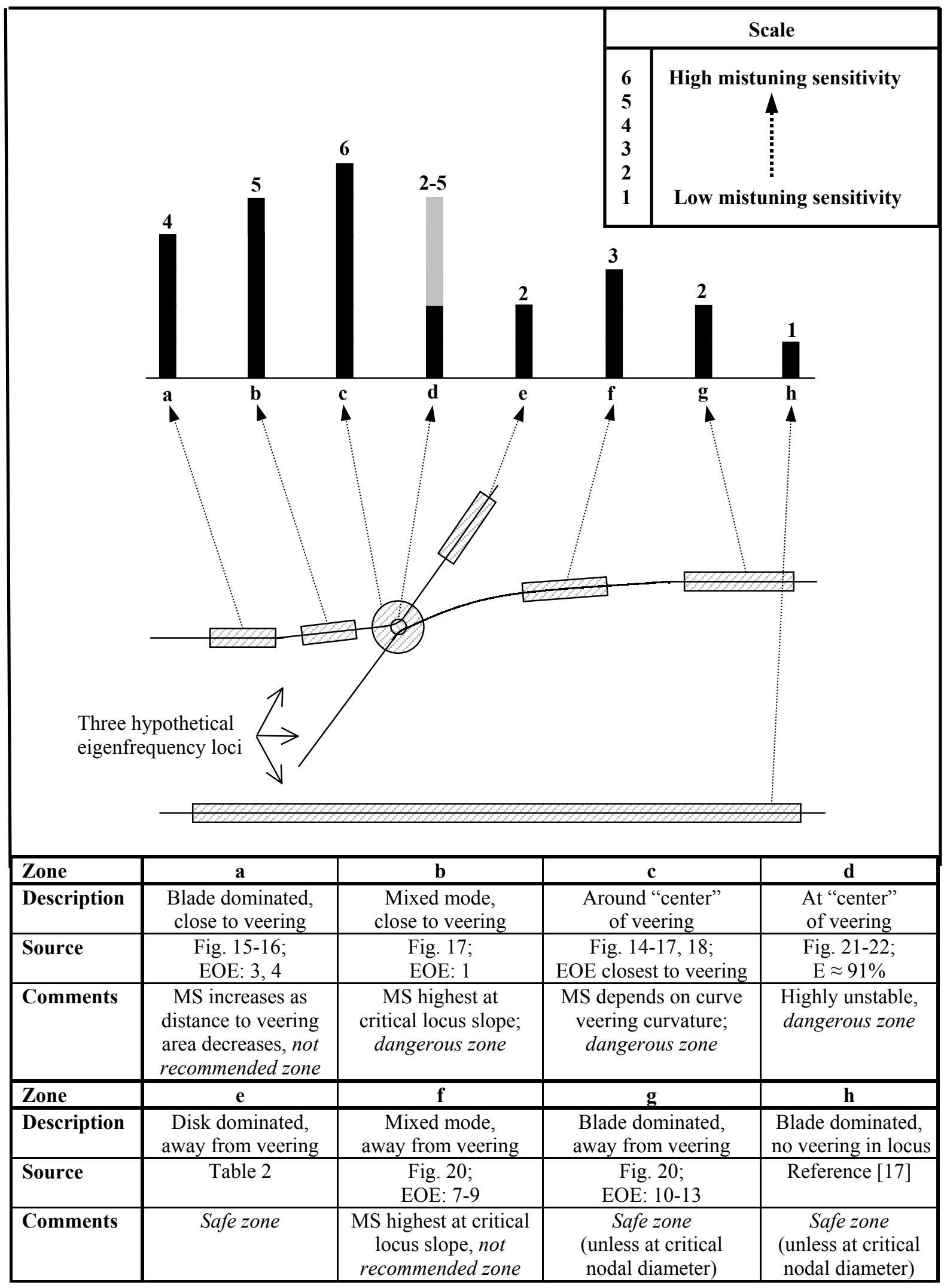

Figure 24. Schematic diagram classifying the various operation zones and the corresponding "anticipated" mistuning sensitivity, with reference to source(s) of information, and comments on operational safety. The assessments are based on the results presented in this study, and may qualitatively differ for other cases. 